\title{
Minimal self y las teorías de la identidad personal en fenomenología
}

Minimal self and phenomenological identity theories

\author{
Agata Joanna Bąk \\ Universidad Autónoma del Estado de México \\ ajbak@uemex.mx
}

DOI: https://doi.org/10.15366/bp2021.28.002

Bajo Palabra. II Época. No28. Pgs: 47-60

Orcid: 0000-0003-1647-5564 
Recibido: 20/09/2021

Aceptado: 12/11/2021

\author{
El presente artículo es fruto de mi participación en el proyecto de investigación \\ Institución y Constitución de la Individualidad: Aspectos Ontológicos, Sociales y de Derecho \\ (PID2020-117413GA-I00 / AEI / 10.13039 / 501100011033) financiado por el \\ Ministerio de Ciencia e Innovación de Espańa.
}

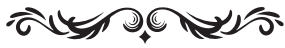

\section{Resumen}

Este artículo investiga la relevancia que puede tener la noción de minimal self, defendida por ejemplo por Zahavi, para una teoría fenomenológica de la identidad que aquí se analiza al hilo del fenómeno de la vejez. Primero, analizo la noción de minimal self tal y como se plasma en los escritos de Zahavi para analizar los presupuestos y argumentos en los que se sostiene. No obstante, segundo, este planteamiento puede resultar problemático para la descripción de los fenómenos vitales cuyo sentido se constituye intersubjetivamente. Así, fenómenos como la enfermedad, la vejez o el género no solo se asientan en la experiencia solitaria e individual, sino que solo pueden ser comprendidos por el propio sujeto en interacción con los demás. El argumento que se aporta, en tercer lugar, para defender este aspecto de la noción de minimal selfes una descripción del fenómeno de la vejez en términos del descubrimiento de una misma como anciana, a partir de los escritos de Simone de Beauvoir.

Palabras clave: minimal self, fenomenologia, teoría de la identidad, personalidad, vejez.

\section{Abstract}

This paper focuses on the relevance that the notion of minimal self, as proposed e.g. by Zahavi, for a phenomenological theory of identity. I will do so by analyzing the phenomenon of coming of age. Firstly, I characterize the notion of minimal self as expressed in $\mathrm{Za}$ havi's claims with a view to analyzing premises and arguments on which it is grounded. Nevertheless, secondly, defending minimal self might pose challenges when it comes to characterize certain life experiences that seem to be constituted intersubjectively. In particular, phenomena such as illness, age or gender are not exclusively constituted in individual, solitary experience, but rather they can only be ultimately understood by the very subject in intersubjective perspective. The argument I offer in the third step of my exposition, is the description of advanced age in terms of personal discovery of being old, drawing mainly on Simone de Beauvoir's writings.

Keywords: Minimal self, phenomenolo$g y$, identity theories, personality, old age. 
A Martín, por cuya culpa o mérito recorrí el camino desde un yo mínimo hasta el yo personal

\section{La noción de minimal self en el debate contemporáneo}

La noción del yo mínimo (minimal self) es la continuación de un perenne debate filosófico acerca de la conciencia. A grandes rasgos, los defensores de minimal self abogan que para hablar de la conciencia o del yo es preciso apelar a un sentido más básico de la subjetividad de la experiencia. Esta tesis puede a su vez modularse en distintos sentidos: se puede afirmar, como parece hacerlo Kriegel (2004), una autorrepresentación de carácter cuasiobjetivo o, como defiende Zahavi (2019a), como una propiedad del flujo de la experiencia consistente en su inherente "what-is-likeness" ${ }^{1}$ para mí. En lo que sigue, me atendré a la exposición que Zahavi hace de la noción de minimal self.

La defensa de esta posición implica varios compromisos filosóficos. En primer lugar, como observa Zahavi (2019a), es un compromiso en cierto sentido antihumeano, en tanto implica pensar en que el sujeto consciente de sus propios actos no es una ilusión edificada meramente en los haces de sensaciones que, por su repetición, se presentan con una pretensión de continuidad sustancial de la identidad subjetiva, persistencia de un yo. Más bien, argumenta Zahavi, el problema debe enfocarse en el hecho de que nunca se trata meramente de sensaciones, sino de experiencias que en su propia estructura exhiben la propiedad de "para mí" (for-me-ness) de cierta cualidad experimentada. En adición, esta experiencia no es un pensamiento reflexivo, en el que a posteriori se atribuyen cierta conciencia de lo ocurrido (como cuando afirmamos "cuando olía aquel intenso perfume, fui yo la que lo olía"); sino que esta conciencia del "para" es presente en el acto mismo de oler. En suma, los estados son de por sí conscientes (aware). El segundo paso para afirmar la consistencia del planteamiento de un yo es afirmar la identidad entre ese carácter "para mî" primario de la experiencia con la conciencia que reflexiona sobre

\footnotetext{
${ }^{1}$ Debido a la incertidumbre sobre cómo traducir el término decido dejarlo en original. La traducción más plausible sería aquí "el cómo del aparecer" que, sin embargo, es poco literal y no goza de una tradición que lo respalde.
} 
sí misma. En otras palabras, solo desde este suelo ya subjetivo es posible reflexionar sobre ciertos estados como propios (el carácter de miness que a diferencia de for-meness implica ya una realización más temática del yo como sujeto del acto) frente a otros que no lo son, y de un yo como un sujeto de la experiencia. En este sentido, la noción del yo mínimo no está comprometida con la idea de autoconciencia lingüística; el carácter subjetivo de la experiencia puede extenderse a los infantes o animales (Zahavi, 2017).

En segundo lugar, la noción de minimal selfse compromete con un presupuesto propio de la fenomenología, esto es, con la idea de que la descripción de la estructura de la experiencia es un punto de partida válido en la filosofía. Más que de los análisis conceptuales, autores como Zahavi siguen el lema husserliano de que "todo lo que se nos ofrece en la intuición originariamente dadora es fuente legítima del conocimiento; que todo lo que se nos ofrece en la "intuición" originariamente (por así decirlo en su realidad en persona) hay que aceptarlo simplemente como lo que se da, pero solo dentro de los límites en que ella se da" (Husserl, 2013:129). En este sentido, el tipo de argumentación que se ofrece y también algunos problemas filosóficos que surgen de esta consideración (véase la sección segunda) están permeados por la idea de la validez de la experiencia como punto de partida.

En tercer lugar, el compromiso implícito -que es realmente compromiso implícito de toda labor filosófica- que se comparte es que la noción de minimal self es un postulado que marca la diferencia en consideración de fenómenos concretos. Pocos filósofos contemporáneos han mostrado consecuencia con este mandato como lo hace Zahavi. El pensador danés se volcó con la cuestión de investigación cualitativa, donde no solo debate los supuestos teóricos, ${ }^{2}$ sino que también incide en problemas concretos relacionados con la experiencia vivida de algunas enfermedades (por ejemplo, Zahavi, 2020), ofreciendo pautas para pensar ciertos problemas éticos involucrados. Además, la noción de minimal self ha sido bien acogida en algunas corrientes de psiquiatría, como Sass y Parnas (2001), por ejemplo, quienes trabajan conjuntamente con Zahavi en el tema de la relación entre fenomenología y psicopatología; y también puede hallarse en los trabajos de Ratcliffe (2017a) o, salvando las distancias, Fuchs (2017) y se encuentra en el corazón de los planteamientos que analizan ciertas dolencias mentales a través de la ruptura o trastorno de la experiencia a estos niveles ínfimos de la vida egoica.

A partir de los textos de Zahavi podemos caracterizar esta noción de la siguiente forma:

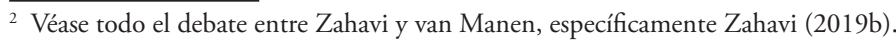


En un primer momento, tenemos que contemplar la noción de minimal self como una forma más primitiva y más fundamental que la forma reflexiva de la autoconciencia (Zahavi, 1999, 2018). Por tanto, no es fácil identificarlo sin más con un quale, un datum de la experiencia, en el sentido "acusativo" (2018:715): ${ }^{3}$ en la experiencia de un objeto yo tengo una experiencia adicional de mí experimentándolo, y sin embargo esa experiencia es ya inherentemente para mí. Más bien el objeto se me aparece a mí: "Ser consciente de las experiencias de una cuando ocurren no es equivalente a ser consciente de una misma como un objeto (secundario), ni tampoco equivalente a ser temáticamente consciente de las experiencias como propias [one's own]" (2018:706). Zahavi insiste en que la pregunta adecuada no es qué es el "yo mínimo", sino más bien "cómo se me da" (2017:194).

Esta experiencia no es, además, en segundo lugar, un fenómeno puntual. La fenomenología, más que de estados mentales, prefiere hablar de la vida anímica, un entramado de percepciones, emociones, valoraciones, acciones a fin de cuentas que están atravesadas por esta vivencia de "para mí". Permea no solo actos a los que presto atención sino en general, la totalidad de fenómenos (dolor de la cabeza, manera de moverse, el sentimiento de encontrarse bien que no se hace explícito pero que me "entona"). En este sentido, el "yo mínimo" se "derrama" más allá del puro presente, exhibiendo rasgos de la durabilidad.

Por tanto, en tercer lugar, más que una operación inferencial posterior al hecho de la experiencia, el minimal self es un carácter integrante [built-in] de la vida de la experiencia (Zahavi, 2017:193-200). Podemos equipararlo con la noción de "for-me-ness" - el carácter del "para mî" de las experiencias, que posibilita en el transcurso de la vida consciente, cuando esta se vuelve consciente de sí, hablar de la propiedad de las experiencias.

A continuación, me gustaría indagar en dos aspectos fenomenológicos, dos debates más bien, que subyacen y moldean la teoría del "yo mínimo”.

\section{El trasfondo y problemas fenomenológicos de minimal self para el desarrollo de una teoría de la identidad}

Podemos observar que la noción de minimal self defiende el carácter irreductible de la subjetividad de los fenómenos de la experiencia. Este modo de pensar se retrotrae a las ideas de Edmund Husserl, para el que las descripciones fenomenológicas

3 Si no se indica lo contrario, las traducciones son de la autora. 
trascurren en un necesario "a priori de correlación" (2008:200): la idea de que en la experiencia de cada cosa, si se analiza el hecho de la experiencia fenomenológicamente, se descubre la actividad de la conciencia para la que la cosa aparece. Ahora bien, la forma en la que Husserl aborda el problema metodológicamente, esto es, a través de una serie de las reducciones que llevan a un yo trascendental que es capaz de volver la mirada sobre lo que se manifiesta, ha despertado y sigue despertando muchas controversias. Zahavi, lector perspicaz de Husserl, sabe bien el problema de la "anonimato" de la vida prerreflexiva, esa vida consciente en la experiencia, minimal self. Por eso mismo, como vimos antes, insiste en la identidad entre el flujo subjetivo de la experiencia y el yo que reflexiona sobre ella. Tiene que haber una identidad entre ambos para que la respuesta fenomenológica al problema sea válida y posible.

Tal vez sea el trabajo de Elisabeth Behnke (2016) el que explica de forma más clara esta aporía. Si se toma en serio la tradición interpretativa que conciba la "intencionalidad operante" (uno de los términos con los que Husserl describe la experiencia ordinaria de las cosas en las que no asumimos conscientemente que somos "nosotros" los sujetos de la experiencia) como un cúmulo de procesos inconscientes, la idea misma de la identidad entre quien reflexione sobre ellas y los procesos corre peligro. Por eso, es necesario conceptualizarlo de otra forma:

Esta tradición interpretativa culmina con el postulado de que, a fin de cuentas, la intencionalidad operante es esencial e inevitablemente anónima, eludiendo en principio el desvelamiento fenomenológico. Pero si de verdad hay una anonimidad impenetrable en el corazón de la vida consciente, esto amenaza el proyecto mismo de la fenomenología basado en la dación de lo que hay que investigar, ya que las estructuras profundas de la experiencia permanecerían inaccesibles, y su anonimidad ineluctable cerraría toda posibilidad de una dación de sí evidencial. Esto llevaría al postulado de Landgrebe de que la reflexión fenomenológica sencillamente no puede alcanzar las dimensiones de la profundidad a lo que aspira. (Behnke, 2016:31-32)

Para poder hablar pues de los análisis de la experiencia de la forma que hace Zahavi, el requisito es que de hecho exista una forma mínima de la conciencia que permita afirmar la validez de las descripciones. ${ }^{4}$ Y como insiste (2017:194), el "yo mínimo" no es ni inconsciente ni anónimo, sencillamente prerreflexivo y fundamentalmente mío, personal, desde su comienzo. "Si la experiencia es en el fondo consciente, pero impersonal, ¿̨cómo podríamos entonces preservar la veracidad de

\footnotetext{
${ }^{4} \mathrm{Si}$ esto incurre o no en una especie de círculo vicioso es algo sujeto a debate. En defensa de Zahavi podemos traer a colación su insistencia en que no describe el "qué", usurpando pretensiones ontológicas sino el "cómo": el cómo equivale a una descripción y no a una explicación. Cfr. p. ej. Zahavi (2017:716).
} 
los informes en primera persona? Decir 'tengo un dolor de cabeza' más bien que 'hay un dolor de cabeza' o que sería demasiado, significaría más bien falsificar antes que articular la experiencia en cuestión" (2018:716). Lo mismo es válido para la distinción entre mis estados y estados de otra persona.

Esta consideración nos lleva a la segunda cuestión fenomenológica que Zahavi aborda en múltiples escritos (por ejemplo, 2017, 2020). El inapelable carácter subjetivo de la experiencia que nos lleva a tomarnos cuenta de nosotros mismos implica que la filosofía se hace en primera persona. En efecto, fenomenología se yergue como un método que parte del esclarecimiento de las estructuras subjetivo-objetivas de la experiencia. Desde esta perspectiva, resulta problemático el papel de los demás en la formación de la identidad personal. Claramente, la hipótesis del yo mínimo compromete con el carácter fuertemente subjetivo y por tanto rechaza las teorías constructivistas según las que el yo sería totalmente construido en las interacciones sociales, y, por tanto, derivado, o bien sería meramente ilusorio. Pero incluso entre las posiciones afines a la teoría fenomenológica se pueden encontrar intentos de articulación de la constitución intersubjetiva del yo que el filósofo danés encuentra inaceptables. Tal sería el caso de Ratcliffe (2017b), contra cuyo planteamiento responde Zahavi en "Thin, thinner, thinnest". Según Zahavi (2017), Ratcliffe va demasiado lejos al proponer que la noción del yo mínimo se constituye intersubjetivamente. Aduce el argumento de los recién nacidos, cuyas primeras experiencias, como dolor o estrés no están mediadas intersubjetivamente. Mientras Zahavi no negaría la idea de la comunidad de los co-constituyentes del sentido del mundo ${ }^{5}$ incluso de lo que soy como persona, jamás podrá negar el carácter subjetivo de esta experiencia. Por ejemplo, y anticipando la próxima sección: el fenómeno de la vejez es algo que implica tanto momentos que son subjetivos) como sensación de cansancio, menor capacidad del movimiento, otra perspectiva temporal), como objetivos (por ejemplo, el hecho de que me vean arrugada, o que no participe en ciertas actividades como antes). La experiencia de los mismos es ineludiblemente propia y debe analizarse desde esta perspectiva.

En este sentido, la teoría del yo mínimo se presenta como una propuesta metodológicamente atractiva. No obstante, para que pueda servir de base para una teoría de la identidad debe desgranar el problema que penetra el corazón de la fenomenología: esto es, en qué sentido puedo ser el sujeto para el mundo, el sujeto de la experiencia, y el objeto en él, para los demás, situado, con unas determinaciones culturales específicas, de género determinado...

\footnotetext{
5 La idea de que los demás son un vínculo necesario para la constitución del mundo objetivo, pero que no constituyen el momento apodíctico de la experiencia del para mí, es algo común para filósofos de orientación husserliana.
} 
En lo que sigue, quisiera profundizar en esta paradoja, trayendo a colación, en primer lugar, un ejemplo que me permitirán desarrollar el trascurso del proceso identitativo.

\section{Llegar a la edad. Análisis fenomenológico del fenómeno de la vejez}

En lo que sigue, abandono la interpretación de la teoría zahaviana de minimal self para ensayar un esbozo de aproximación a la teoría de la identidad a través del fenómeno de la vejez. ${ }^{6}$ Lo tomo a modo de un ejemplo, esto es, a través de la descripción de la edad madura pretendo investigar cómo en ella operan las nociones y se presentan los problemas antes anunciados.

El fenómeno de la vejez ha sido objeto del análisis fenomenológico. El propio Husserl menciona de pasada la posibilidad o la necesidad de este tipo de investigación: "El yo espiritual [persona, explicación propia] puede ser así aprehendido como un organismo, un organismo de capacidades de su desarrollo en un estilo normal típico con los niveles de infancia, juventud, madurez, vejez" (2005:254). Autoras como de Beauvoir (1996), Heinämaa (2014) o Venebra (en prensa) se han centrado en distintos aspectos del fenómeno de la vejez. Así, la autora francesa describe en su obra La vejez $^{7}$ la experiencia vivida de la edad avanzada. En un exquisito volumen dedicado a este libro (Stoller, 2014), varias filósofas reflexionan sobre la trascendencia de este estudio para la descripción fenomenológica de lo implícito en ella. De la misma forma, una puede adoptar un enfoque estrictamente fenomenológico para analizar un aspecto de la vejez, como por ejemplo la transformación de los horizontes temporales o corporales (Aurenque, 2021, Venebra, en prensa) o aspectos de carácter político, ético o social (por ejemplo, Heinämaa, 2014). La literatura es inmensa e interseccional, cruzando los problemas de edad, raza y género. ${ }^{8}$ En lo que sigue, me centraré en el análisis del proceso de descripción fenomenológica de la identidad de la persona mayor para dilucidar el dilema antes anunciado. Lo haré teniendo en cuenta el análisis de Heinämaa (2014).

En su libro La vejez, Simone de Beauvoir describe este fenómeno vital de forma fenomenológica. Me centraré en el capítulo quinto, en el que la filósofa aborda la

\footnotetext{
${ }^{6}$ Una versión incipiente de este ejercicio fue presentada en el congreso Lo inquietante son los otros en la Universidad de Sevilla bajo el título "Lo inquietante de la vejez. La mirada del otro en la configuración del estilo personal", el pasado noviembre de 2019.

7 Para propósitos de este artículo citaré la edición inglesa The coming of age (de Beauvoir, 1996).

${ }^{8}$ Un estudio fenomenológicamente esclarecedor al respecto es el de Wehrle (2020) y, en general, el libro entero (Schweda, Coors y Bozzaro, eds. 2020).
} 
cuestión desde perspectiva fenomenológica. En los capítulos anteriores, de Beauvoir analiza los datos etnográficos, antropológicos y sociales de la vejez en distintas sociedades. ${ }^{9}$ La descripción que da la filósofa del fenómeno comienza con una palabra que denota el marcado -si bien ambiguo- carácter subjetivo del fenómeno de la vejez: el descubrimiento. Mi tesis en este punto es que a través de su análisis del descubrimiento podemos trazar el paradójico engranaje sujeto de mi experiencia. pero también un objeto para los demás. ${ }^{10}$ Es la paradoja entre el yo, la primera persona con su vida egoica, con sus experiencias y "un yo" envejecido, del que no se es consciente, que tiene que ser asumido ante signos, miradas y evidencias provenientes desde la mirada de los demás. La vejez es eso: un descubrimiento que hay que asumir; un acontecimiento que empieza en algún lugar del cuerpo y que se resume en esta aporética constatación: "Hay dentro de mí un Otro, esto es, la persona que soy para el que me ve desde fuera, [y esta] es vieja: y este otro soy yo" (1996:284). Sugiero que este movimiento de asunción de algo que me pasa es crucial para la construcción de una teoría de la identidad.

Sigamos con el ejemplo de la filósofa. En un primer lugar, si realmente hay ese otro en nosotros que es mayor, pero del que no nos percatamos, es natural que la revelación de la edad venga a nosotros desde fuera: desde los otros (de Beauvoir, 1996:288). Estos otros pueden ser los demás o tal vez puede ser el espejo (véase el caso de Lou von Salomé citado en el libro). Mi ser es por tanto no solo algo que yo experimento en la intimidad de mi cuerpo vivido, sobre el que impero; también es un ser mundano, entre otros seres animados que salen a mi encuentro. En este caso concreto, los animales son además personas, y tienen nombres y comprenden signos: llaman la atención sobre ciertos aspectos: las manos arrugadas, la caída del pelo, quedan tipificadas como indicios de una cierta edad. Mi materialidad, que no experimento de forma inmediata como nos insta a considerar la actitud natural, de repente pasa a ser el foco de la atención: sobresalen ciertos aspectos suyos, y eso porque han sido reflejados en los demás con una significación. Se crea una imagen, "genérica, contradictoria y vaga" (1996:291) en un esfuerzo de verme como los demás quieren verme a mí.

En este caso, se desprende del análisis de Beauvoir que la "edad" es algo revelado para mí, no necesariamente algo que se vive de forma evidente ("La edad no se

\footnotetext{
9 Varias autoras, entre ellas Heinama (2014) ponen de relieve la similitud estructural entre La vejez y El Segundo sexo. El pararelismo entre ambas obras puede ser indicativo del modo en que la filósofa francesa comprende el método filosófico, lo cual hace más interesante el intento de profundizar en él en este estudio.

${ }^{10}$ Usar en este contexto la palabra "objeto" no debe tener connotaciones deshumanizantes. Como afirman Heinämaa y Jardine, en cierto sentido toda percepción del otro ser humano tiene un carácter objetivante. "Se mienta en algún modo de ser" (2021:19) delante de mis ojos. Este modo de percibir no tiene por qué implicar la negación del carácter personal, psíquico y de valor de otra persona.
} 
experimenta en el modo del 'para sí y no tenemos de él el mismo conocimiento lúcido como es el caso del cogito", 1996:292). De hecho, y este es el segundo apunte, la vivencia íntima y corporal de la edad no tiene por qué corresponderse con la visión de los demás. A diferencia de experiencias que rompen o transforman de forma aguda el curso de la experiencia, como puede ser la enfermedad, la vejez puede ser meramente "una cierta dificultad de ser" (ib.). Con todo, la visión del sujeto envejecido que proporciona de Beauvoir es casi desgarradora: una cosa soy yo en la intimidad, en la que impero, y otra, muy distinta es la trascendencia del ego en la situación fáctica, situada, entre los demás. "La vejez" -escribe de Beauvoir- "es algo más allá de mi vida, algo fuera. Algo de lo que no puedo tener ninguna experiencia plena e interna" (1996:291).

Esta descripción podría chocar con lo antes constatado desde el planteamiento de Zahavi. Si el análisis beauvoiriano se toma en su sentido fuerte, parecería que el yo mínimo sirve de poco para analizar los fenómenos vitales, que es inútil para la teoría de la identidad ya que en última instancia se trata de situaciones que desbordan al sujeto.

En lo que sigue procuraré reconducir esta aparente paradoja a una descripción más plausible. La intuición básica es que la noción del yo mínimo es un elemento inalienable en el desarrollo de la teoría de la identidad (al fin y al cabo, es $m i$ identidad), pero su carácter apodíctico no significa que la vida personal se reduzca a algo subjetivo en un sentido fuerte: la experiencia para mí exhibe momentos que son distintos a mí. No me creo, por así decirlo, desde la nada.

Así, argumento que existen dos esferas que hay tener en cuenta en la constitución de la identidad personal en relación con los demás: una pasiva y otra activa. La forma en la que puedo describir la identidad personal es a través de un movimiento de hacerme persona. ${ }^{11} \mathrm{Y}$ esto implica justamente este carácter de descubrimiento e incluso, como quiere Heinämaa, de transformación. En este sentido, me constituyo como persona en cada acto -bien reflexivo, de comprenderme como tal, bien a nivel prerreflexivo, viviendo el mundo a partir de la razón, con la riqueza de sentidos culturales y anímicos que me son disponibles.

En efecto, muchas veces algunos fenómenos vitales pasan desapercibidos hasta que llaman nuestra atención a través de la mirada del otro. De Beauvoir detalla con

\footnotetext{
11 Esta noción está inspirada sobre todo en el trabajo de Behnke (1997). En este artículo la autora expone la idea de making a body, esto es, hacerme un cuerpo. Propone partir de la concreción de una experiencia - para ella, es la postura erguida, para el caso de este trabajo, es la vejez - para ir desplegando todos los estratos involucrados en la misma. En este sentido, el modo de describir la identidad humana consistiría en atender al "transcurrente [ongoing] 'cómo' de nuestra vida corporal/intercorporal que experimenta el mundo”. (1997:181) Aunque no está claro si para la autora este es el proceso de la descripción de la identidad, nos tomamos la licencia de aprovechar este pensamiento para el argumento que nos concierne.
} 
grandilocuencia ejemplos en los que las personas se han descubierto mayores. En la mayoría de los casos ha sido una percatación dolorosa.

Este momento en el que un elemento de mi propia identidad aparece instituido y casi imprimido por otro en mí es el momento pasivo de la experiencia. Husserl reconoce claramente esta influencia del otro en la configuración del yo:

El desarrollo de una personalidad está determinado por la influencia de otra, por la influencia de pensamientos ajenos, de sentimientos ajenos sugeridos, de ordenes ajenas. La influencia determina el desarrollo personal, sepa o no la persona misma más tarde algo sobre ello, se acuerde o no de ello, sea o no capaz de determinar ella misma el grado y la índole de la influencia. (Husserl, 2005:316).

El desconocimiento de esta determinación parece algo confuso para una teoría que priorice la experiencia en primera persona. No obstante, tal vez la distinción más importante para el caso sea la establecida por Husserl en el mismo texto: "Tenemos por tanto que distinguir entre la persona humana, la unidad aperceptiva que captamos en la percepción de sí mismo y en la percepción de otro, y la persona como el sujeto de los actos de razón (...)" (2005:317). ${ }^{12}$

En este caso, ¿`cómo se constituye la identidad humana? La suposición tácita que aquí manejo es que la cuestión de la identidad personal no se juega en el binomio ser (persona) - tener (cierta personalidad o identidad); más bien se trata de reconstruir el gesto de hacerse persona. Esta observación se sostiene en el hecho de que la vida personal, más que enclaustrarse en una identidad oronda, es un fluir, una dinámica entre lo ya logrado y los planes y proyectos. Al respecto, dice Brudzinska:

La vida de la persona trascendental no es vida de mera identidad, sino más bien una vida dinámica de individuación. Semejante individuación no tiene lugar en un mundo abstracto de meras cosas o tesis cientificas, sino más bien en un mundo de la vida concreto, en tanto que mundo, dado sensual e intuitivamente, de nuestras necesidades experimentadas y expresadas corporalmente, de nuestros deseos, planes y objetivos prácticos, como el mundo en el que nos encontramos entre otros (2014:91, cursivas en el original).

De la misma forma que nuestro cuerpo no tiene una postura fija o una única forma básica, sino que se actualiza en cada gesto y también en la reflexión sobre la postura y trabajo con las habitualidades por ejemplo en el entrenamiento; de la misma forma la persona también se hace, se teje entre las experiencias propias y las miradas de los demás. Pero es justo en la capacidad de descubrirse de una forma u

\footnotetext{
${ }^{12}$ La razón en este contexto no se limita a una esfera meramente teórica y reflexiva, sino a la total estructura de la vida consciente. Los actos de la razón serán, por tanto, todos los actos efectuados por la conciencia.
} 
otra y en la toma de las decisiones o asunciones está el punto álgido de este proceso. Es cuando la identidad personal en proceso se reafirma a través de sus actos. De esta forma, el carácter del para mí de la experiencia, ese yo mínimo postulado por Zahavi se mantiene en la totalidad de la vida subjetiva. Esto no contradice la idea de que ciertos caracteres me sean atribuibles desde fuera. No obstante, la verdadera apuesta fenomenológica a favor de la que he intentado argumentar, consiste en mantener que lo crucial no es que todas las atribuciones se hagan subjetivamente, sino que el modo de describirlas implique necesariamente una referencia a la persona que lo experimenta.

\section{Conclusiones}

En este trabajo he procurado explorar la noción de minimal self propuesta por Zahavi. He explicado que su mayor apuesta consiste en afirmar la necesaria dimensión consciente de todo acto de la experiencia sin que ello implique sustantivar tal conciencia. Esta más bien se ve como el modo "para mî", prelingüístico y prerreflexivo. Esta característica de la conciencia, sugería, es el punto de anclaje necesario para poder atribuirse estados mentales, para tomar conciencia de algo como una experiencia propia. Tras examinar ciertos compromisos con la tradición fenomenológica involucrados en esta postura, he sugerido que la idea constructivista de la identidad personal debe contrastarse con la que pueda ofrecer una teoría de la identidad basada en minimal self. Para ello, he retomado la reflexión beauvoiriana sobre la vejez mostrando cómo en ella intervienen vivencias de la propia corporalidad o temporalidad, pero también la mirada ajena. Finalmente, en un ejercicio fenomenológico he intentado reconducir lo "ajeno" a la experiencia de una misma en el mundo. Esto me ha llevado a sugerir que la forma correcta de hablar de la identidad personal no es tanto "qué" es la persona sino "cómo" se descubre o qué elementos implica. Esta posición no está exenta de objeciones y ha de precisarse en futuras investigaciones. 


\section{REFERENCIAS BibLIOGRÁFICAS}

Aurenque Stephan, D. (2020). Fenomenología de la vejez y el cuerpo como anclaje al tiempo: "Se debe ser viejo para reconocer lo breve que es la vida", Valenciana, 14(27), 2021, 147-168. DOI: https://doi.org/10.15174/rv.v13i27.479

Behnke, E. (1997). Ghost Gestures: Phenomenological Investigations of Bodily Micromovements and Their Intercorporeal Implications. Human Studies 20, 1997, pp. 181-201. DOI: https://doi.org /10.1023/a:1005372501258

Behnke, E. (2016). La vida operativa: the transcendental disclosure of operatively functioning life. Acta Mexicana De Fenomenologia, 1, 2016, pp. 29-48.

De Beauvoir, S. (1996). The coming of age. W. W. Norton \& Company.

Brudzinska J. (2014). Becoming a Person in a Life-World, Paradigmi. Rivista di critica filosofica, 3, 2014, pp. 91-110. DOI: https://doi.org/10.3280/ PARA2014-003007

Fuchs, Th. (2017). Self across time: the diachronic unity of bodily existence, Phenom. Cogn Sci, 16, pp. 291-315 DOI: https://doi.org/10.1007/s11097-0159449-4

Heinämaa, S. (2014). Transformations of Old Age. Selfhood, Normativity, and Time, en S. Stoller (ed.) Simone de Beauvoir's Philosophy of Age. De Gruyter, 2014, pp. 167-187.

Heinämaa, S. y Jardine, J. (2021). Objetivación, inferiorización y proyección en la investigación fenomenológica acerca de la deshumanización, en A. Bak, (ed.) Las fronteras del sentido. Filosofia y critica de la violencia. UAEMEX/Sb Editorial, pp. 15-40.

Husserl, E. (2005). Ideas para una fenomenología pura y una filosofía fenomenológica. Libro Segundo: Investigaciones fenomenológicas sobre la constitución. Fondo de Cultura Económica.

Husserl, E. (2008), La crisis de las ciencias europeas y la fenomenología trascendental. Prometeo.

Husserl, E. (2013). Ideas relativas a una fenomenología pura y una filosofía fenomenológica. Libro primero: Introducción general a una fenomenología pura. Fondo de Cultura Económica.

Kriegel, U. (2004). Consciousness and Self-Consciousness, Monist, 87 (2), $182-$ 205. DOI: https://doi.org/10.5840/monist20048725

Parnas, J. y Sass, L. A. (2001). Self, Solipsism, and Schizophrenic Delusions, Philosophy, Psychiatry, \& Psychology, 8 (2/3), 2001, pp. 101-120. DOI: https:// doi.org/10.1353/ppp.2001.0014 
Ratcliffe, M. (2017a). Real Hallucinations: Psychiatric Illness, Intentionality, and the Interpersonal World. The MIT Press.

Ratcliffe, M. (2017b). Selfhood, Schizophrenia, and the Interpersonal Regulation of Experience en Durt, Fuchs y Tewes (eds.) Embodiment, enaction and culture. Investigating the constitution of the Shared World. The MIT Press.

Stoller, S. (ed.) (2014). Simone de Beauvoir's Philosophy of Age. Gender, Ethics and Time. Gruyter.

Schweda, M., Coors, M. y Bozzaro, C. (eds.) (2020). Aging and Human Nature. Perspectives from Philosophical, Theological, and Historical Anthropology, Springer.

Venebra, M. (en prensa). El tiempo del cuerpo. La vejez. Fenomenología de la fragilidad y el desgaste, aceptado para publicación en Areté.

Wehrle, M. (2020). Becoming Old. The Gendered Body and the Experience of Aging, en Schweda, Coors y Bozzaro (eds.) Aging and Human Nature. Perspectives from Philosophical, Theological, and Historical Anthropology. Springer, pp. 75-95.

Zahavi D. (1999). Self-awareness and Alterity. A Phenomenological Investigation, Nortwestern University Press.

Zahavi, D. (2017). Thin, Thinner, Thinniest: Defining the Minimal Self, en Durt, Fuchs y Tewes (eds.) Embodiment, enaction and culture. Investigating the constitution of the Shared World. The MIT Press, pp. 193-200.

Zahavi, D. (2018). Consciousness, Self-Consciousness, Selfhood: a Reply to some Critics, Rev. Phil. Psych. 9, 703-718, 2018. DOI: https://doi.org/10.1007/ s13164-018-0403-6

Zahavi, D. (2019a). Consciousness and (minimal) selfhood: Getting clearer on for-me-ness and mineness, en Kriegel (ed.) The Oxford Handbook of the Philosophy of Consciousness. Oxford University Press, pp. 635-653.

Zahavi, D. (2019b). Getting it quite wrong. Van Manen and Smith on Phenomenology, Qualitative Health Research, 29 (6), 2019, pp. 900-907. DOI: https://doi.org/10.1177/1049732318817547

Zahavi, D. (2020). Locked-In Syndrome: a Challenge to Standard Accounts of Selfhood and Personhood? Neuroethics 13, pp. 221-228. DOI: https://doi. org/10.1007/s12152-019-09405-8

DOI: https://doi.org/10.15366/bp2021.28.002

Bajo Palabra. II Época. No28. Pgs: 47-60 